\title{
Exposure Evaluation Method Based on Histogram Statistics
}

\author{
Wen Chen ${ }^{1,2, *}$ and Xinglong $\mathrm{Li}^{1}$ \\ ${ }^{1}$ Shanghai Aerospace Control Technology Institute, shanghai 201109, China \\ ${ }^{2}$ Shanghai Key laboratory aerospace intelligent control technology, China \\ ${ }^{*}$ Corresponding author
}

\begin{abstract}
Aiming at the inadequate or excessive exposure problem existed in the imaging system in a scene where there is no specific background or foreground, this paper presents a histogram-based exposure evaluation method. With analysis of statistics features of histograms in different exposure type images, the third moment about middle gray is used to evaluate the exposure effect. For color images, the histogram is calculated only in the component which holds the largest mean brightness considering of efficiency. Experimental result shows that the proposed method can evaluate the exposure effect without the support of database, and it has low complexity and is easy to implement.
\end{abstract}

Keywords-exposure evaluation;image histogram; third moment; middle gray

\section{INTRODUCTION}

In consumer digital cameras, one of the image pipeline tasks includes auto-exposure (AE). This module provides the right amount of exposure by adjusting the camera exposure in an automatic manner. For auto-adjusting of the camera exposure, we need to make a feedback system that can update the camera exposure according to an evaluation of the current exposure. Therefore the exposure evaluation method is a key point in auto-exposure system.

Basically, there have been two approaches. One approach is based on using a reference brightness value: the longer the exposure time, the brighter the image intensity. Auto-adjusting of camera exposure can be done by comparing current brightness to a certain reference brightness. For example in [1], an image is divided into a matrix of small sub-images and the average brightness in the sub-images is used to set up a reference brightness value. The reference value is then reached by adjusting the aperture size. A similar approach has been adopted for setting shutter speed. Obviously, the application of the average brightness-based method is limited. Instead of simple information (the average brightness), various evaluation methods such as matrix, center-weighted, entropy and gradient have been utilized to set the exposure value based on brightness of the image. In [2-6], a number of relationships and rules relating brightness to exposure have been presented which are basically derived via experimentation.

Another approach adopted by some digital camera manufacturers is to learn the brightness-exposure relationship for different lighting conditions via image example [7-9]. Between the above two approaches, the learning via examples approach provides a more robust solution due to the fact that it incorporates many different lighting conditions and type of photography. However, such an approach requires access to a large database of images captured under different lighting conditions.

Furthermore, the conventional methods are suitable for the scene where both background and foreground are specific and can be distinguished easily. However, for some special scenes where there is no specific background or foreground, such as scenes full of forests, oceans or mountains, the conventional methods do not lead to a proper exposure level.

Image quality and the histogram of an image are closely related. A histogram is a graphical representation of the pixels exposed in an image. In a well-exposed image, the gray distribution is uniform and there is no overflow in both ends of the histogram. And the histogram can be used to determine the mean, variance, minimum, and maximum luminance for an image. Therefore, histogram-based method is used to evaluate the exposure value in [10-15]. However, these papers mainly focus on the exposure in the region of interest. Reference [14] evaluates the exposure effect by judging if there are pixels in both ends of the histogram, which is easily affected by noise.

In order to solve this problem, skewness of the histogram is used to judge the exposure adequacy in this paper. To obtain the skewness of the histogram, a third moment is computed in terms of deviations from the reference brightness instead of from mean. Experimental result shows that the proposed method is valid.

\section{Exposure Evaluation Method BASED ON HISTOGRAM STATISTICS}

Large amount of experiments show that, instead of bimodal, the histogram of the low contrast images is usually unimodal. Meanwhile, the histogram is unimodal for some special scenes where there is no specific background or foreground too. In both cases, it is poor in distinguishing the object from the background. The conventional methods do not lead to a proper exposure level. In this paper, a method based on the third moment is proposed to evaluate the exposure effect of the image whose histogram is unimodal.

\section{A. The Concept of Moments}

Moments are well known for their application in image analysis, since they can be used to derive invariants with 
respect to specific transformation classes. A moment is a specific quantitative measure of the shape of a set of points. If the points represent probability density, then the zeroth moment is the total probability, the first moment is the mean, the second central moment is the variance, and the third moment is the skewness.

The nth moment of a real-valued continuous function $f(x)$ of a random variable $x$ about a value $C$ is

$$
\mu_{n}=\int_{-\infty}^{\infty}(x-C)^{n} f(x) d x
$$

The $n$th moment about zero of a probability density function $f(x)$ is the expected value of $X^{n}$ and is called a raw moment. The moments about its mean (with $C$ being the mean) are called central moments; these describe the shape of the function, independently of translation. For the second and higher moments, the central moments are usually used rather than the moments about zero. A central moment is the expected value of a specified integer power of the deviation of the random variable from the mean instead of from zero; therefore, they provide clearer information about the distribution's shape.

The first central moment $\mu_{1}$ is 0 . The second central moment $\mu_{2}$ is called the variance. The third central moment is used to define the standardized moment which is used to define skewness because it is the measure of the lopsidedness of the distribution. In a symmetric distribution, its third central moment equals zero. A distribution that is skewed to the left (the tail of the distribution is longer on the left) will have a negative skewness. A distribution that is skewed to the right (the tail of the distribution is longer on the right) will have a positive skewness.

\section{B. The Proposed Method}

If the gray distribution is uniform, the dynamic range is high, the contrast is high, and the image is clear. As the gray distribution is uniform, it is symmetric too. As described in section $\mathrm{A}$, in a symmetric distribution, its third central moment equals zero. Meanwhile, the experimental result shows that the skewness of a histogram approaches to zero as the gray distribution becomes more uniform, especially for the images whose histogram is not bimodal. Therefore, the skewness of a histogram is adopted to evaluate the uniformity of a histogram in this paper.

The third central moment is a measurement of skewness of a histogram about mean. However, usually mean brightness does not express the proper exposure. The gray distribution symmetry about the reference brightness, rather than the symmetry about the mean brightness, reflects the exposure accuracy. It reminds us to combine the third moment with a reference brightness value to evaluate the exposure effect.

Most scenes produce an overall brightness of middle gray or $18 \%$ reflection. Hence, in most cases, the use of middle gray luminance generates a proper exposure, which means the middle gray can be used as a reference brightness value. Therefore, in order to evaluate the exposure effect, a method based on the third moment and the middle gray is proposed in this paper.
Let $U$ be an $M \times N$ image. $z_{k} \in\{0,1,2, \cdots, 255\}$ is the gray value of $U$. The histogram of $U, p\left(z_{k}\right)$ is defined as follows:

$$
p\left(z_{k}\right)=q\left(z_{k}\right) /(M \times N), k=0,1, \ldots, 255,
$$

where $q\left(z_{k}\right)$ is the total number of frequencies of the pixels whose gray value is equal to $z_{k}$ in image $U$.

Let $U_{i}$ be the $i$ th frame, and the histogram of $U_{i}, p_{i}\left(z_{k}\right)$ is defined as follows:

$$
p_{i}\left(z_{k}\right)=q_{i}\left(z_{k}\right) /(M \times N), k=0,1, \ldots, 255,
$$

where $q_{i}\left(z_{k}\right)$ is the total number of frequencies of the pixels whose gray value is equal to $z_{k}$ in the $i$ th frame $U_{i}$.

Let $C$ be middle gray, the third moment about $C, \mu$ is defined as follows:

$$
\mu=\sum_{k=0}^{255}\left(z_{k}-C\right)^{3} p\left(z_{k}\right)
$$

Middle gray $\mathrm{C}=128$ in this paper.

For the $i$ th frame $U i$, the third moment about middle gray, $\mu(i)$ is defined as follows:

$$
\mu(i)=\sum_{k=0}^{255}\left(z_{k}-128\right)^{3} p_{i}\left(z_{k}\right)
$$

The third moment about middle gray $\mu(i)$ is employed to evaluate the exposure effect for the $i$ th frame. If $\mu(i)<0$, the distribution is skewed to the left, which means the image is too dark. If $\mu(i)>0$, the distribution is skewed to the right, which means the image is too bright. If $\mu(i)=0$, the distribution is symmetric, which means the exposure is correct. Let $\mathrm{T}_{i}$ be the exposure time of the $i$ th frame. For the frame after the $i$ th frame, its exposure time $\mathrm{T}_{i+1}$ can be adjusted as follows:

$$
\begin{cases}\mathrm{T}_{i+1}=\mathrm{T}_{i}+\Delta \mathrm{T}_{i}, & \text { if } \mu(i)<0 \\ \mathrm{~T}_{i+1}=\mathrm{T}_{i}-\Delta \mathrm{T}_{i}, & \text { if } \mu(i)>0\end{cases}
$$

where $\Delta \mathrm{T}_{i}$ is the exposure time adjustment step.

For color images, it is time-consuming to compute the total amount of histogram information from all color components in each frame. For efficiency, the histogram is calculated only in the component which holds the largest mean brightness.

\section{EXPERIMENTAL RESULT}

In this section, a group of color images, Leaves, are used to validate the performance of the proposed method. Each image has 256-gray levels in all of three channels, 0 is the darkest and 255 is the brightest. All experiments are done under $\mathrm{Vc}++6.0$. Two conventional exposure estimation criteria, the entropy and the mean-value, are used for comparison.

In this experiment, different exposure type images are captured by setting different exposure time in the same scene. 
CMV20000 is used in the experiments. The exposure time can be set from $100 \mu \mathrm{s}$ to $4000 \mu \mathrm{s}$ when the frame frequency is $25 \mathrm{fp} / \mathrm{s}$. Parts of images are shown in Figure I. For the purpose of efficiency, only Green component histogram is participated in calculation because mean brightness of Green component is the largest during Red, Green and Blue components. Figure II shows Green component histograms of the images shown in Figure I. Table I lists the various features of Green component, including the mean value, the entropy, the $3^{\text {rd }}$ moment about mean ( $3^{\text {rd }}$ central moment), and the $3^{\text {rd }}$ moment about middle gray. Pixels in the whole image are participated in the computation.

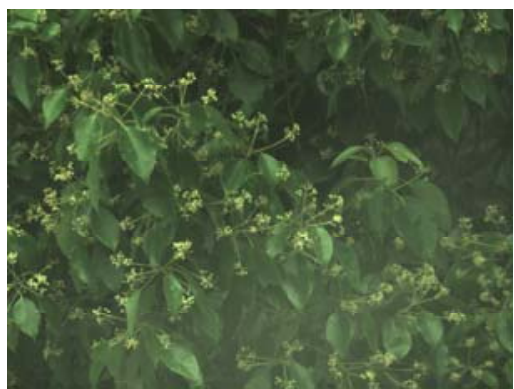

(1) $\mathrm{T}=2000 \mu \mathrm{s}$

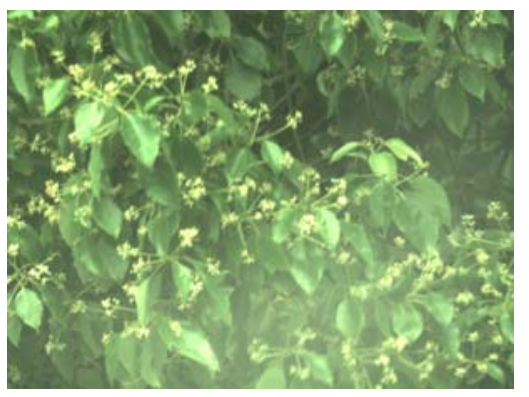

(4) $\mathrm{T}=3500 \mu \mathrm{s}$

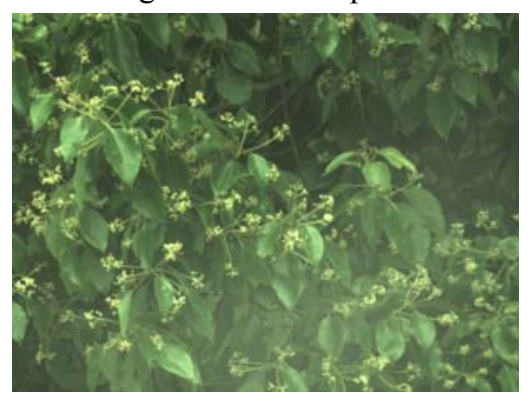

(2) $\mathrm{T}=2596 \mu \mathrm{s}$

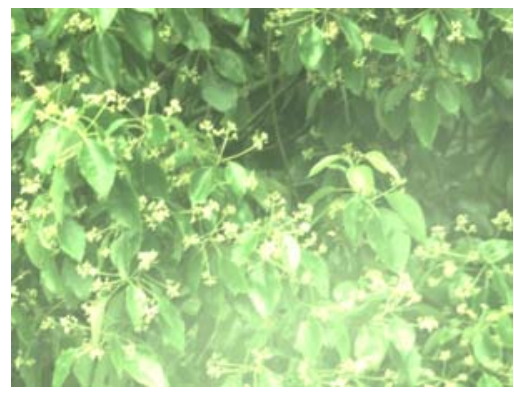

$(5) \mathrm{T}=4500 \mu \mathrm{s}$

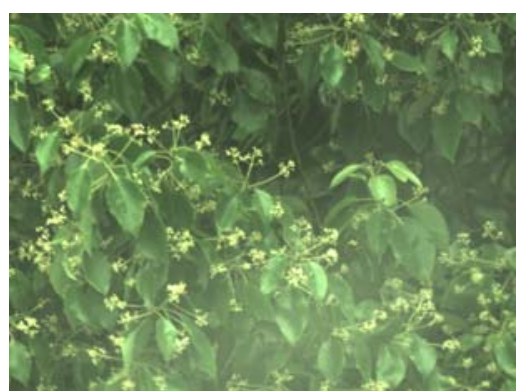

(3) $\mathrm{T}=3100 \mu \mathrm{s}$

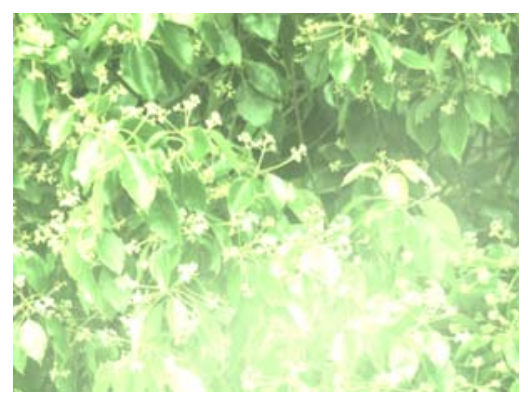

(6) $\mathrm{T}=5500 \mu \mathrm{s}$

FIGURE I. LEAVES CAPTURED IN DIFFERENT EXPOSURE TIME

As shown in Figure I, the first image is very dark. As the exposure time increases, the mean value is getting bigger and bigger (see Table I), and the image is getting brighter and brighter. Figure I(5) and Figure I(6) are obviously overexposed.

TABLE I. EXPERIMENTAL RESULTS FOR LEAVES CAPTURED IN DIFFERENT EXPOSURE TIME

\begin{tabular}{|c|c|c|c|c|c|}
\hline $\begin{array}{c}\text { Frame } \\
\text { number } \\
i\end{array}$ & $\begin{array}{c}\text { Exposure } \\
\text { time } \\
\mathrm{T}_{i}(\mu s)\end{array}$ & $\begin{array}{l}\text { Mean } \\
\text { value }\end{array}$ & Entropy & $\begin{array}{c}3^{\text {rd }} \\
\text { moment } \\
\text { about } \\
\text { mean }\end{array}$ & $\begin{array}{c}3^{r d} \text { moment } \\
\text { about } \\
\text { middle } \\
\text { gray } \mu(i)\end{array}$ \\
\hline 1 & 2000 & 89.165 & 6.6536 & 0.4400 & -1.8183 \\
\hline 2 & 2380 & 99.1397 & 6.7910 & 0.6041 & -1.0230 \\
\hline 3 & 2500 & 106.3812 & 6.9407 & 0.6595 & -0.5960 \\
\hline 4 & 2596 & 114.5455 & 7.0475 & 0.7754 & -0.0529 \\
\hline 5 & 2740 & 121.8217 & 7.1119 & 0.7788 & 0.4022 \\
\hline 6 & 3100 & 132.7647 & 7.1883 & 0.7890 & 1.1781 \\
\hline 7 & 3500 & 153.3941 & 7.2576 & 0.6777 & 3.2415 \\
\hline 8 & 4000 & 171.9556 & 7.1216 & 0.3876 & 6.2640 \\
\hline 9 & 4500 & 191.1391 & 6.7641 & -0.1595 & 10.1888 \\
\hline 10 & 5000 & 206.4181 & 6.0698 & -0.5284 & 14.1927 \\
\hline 11 & 5500 & 219.9274 & 5.1766 & -0.6890 & 18.0179 \\
\hline 12 & 6000 & 232.4526 & 4.1512 & -0.6300 & 22.1286 \\
\hline
\end{tabular}

As shown in Table I, the entropy value climbs to its peak when the exposure time is equal to $3500 \mu \mathrm{s}$. It means that Figure I(4) will be the best exposed image according to the entropy value. Meanwhile, the $3^{\text {rd }}$ moment about mean is closest to zero when the exposure time is equal to $4500 \mu \mathrm{s}$, which means that Figure I(5) will be the best exposed image according to the $3^{\text {rd }}$ moment about mean. However, as shown in Figure I, Figure I(4) and Figure I(5) are obviously overexposed. The mean value reaches to 132.7647 , which is very close to 128 , when the exposure time is equal to $3100 \mu \mathrm{s}$. It means that Figure $\mathrm{I}(3)$ will be the best exposed image according to the mean value. The $3^{\text {rd }}$ moment about middle gray is closest to zero when the exposure time is equal to $2596 \mu \mathrm{s}$, which means that Figure I(2) will be the best exposed image according to the $3^{\text {rd }}$ moment about middle gray. Comparing Figure I(2) with Figure I(3), it is obvious that Figure I(3) is brighter than Figure I(2) and the middle and lower part in Figure I(3) is over-exposed. Furthermore, as shown in Figure II, the histogram of Figure I(3) is more inclined to right and the dynamic range is lower compared with Figure II(3). Therefore, Figure I(2) is better than Figure I(3).

Furthermore, as shown in Figure II, the histograms of Figure I(4), Figure I(5) and Figure I(6) are skewed to the right. The histogram moves from the left to the middle and then to the far-right as the absolute value of the $3^{\text {rd }}$ moment about middle gray goes down and up. Moreover, the dynamic range is higher when the skewness of a histogram approaches to zero. In the meanwhile, there are only green leaves and yellow 
flowers in this test scene, which means that there is neither specific foreground nor background. Thus the histogram is nearly unimodal. According to the result, the $3^{\text {rd }}$ moment about middle gray leads to a better exposure level compared with
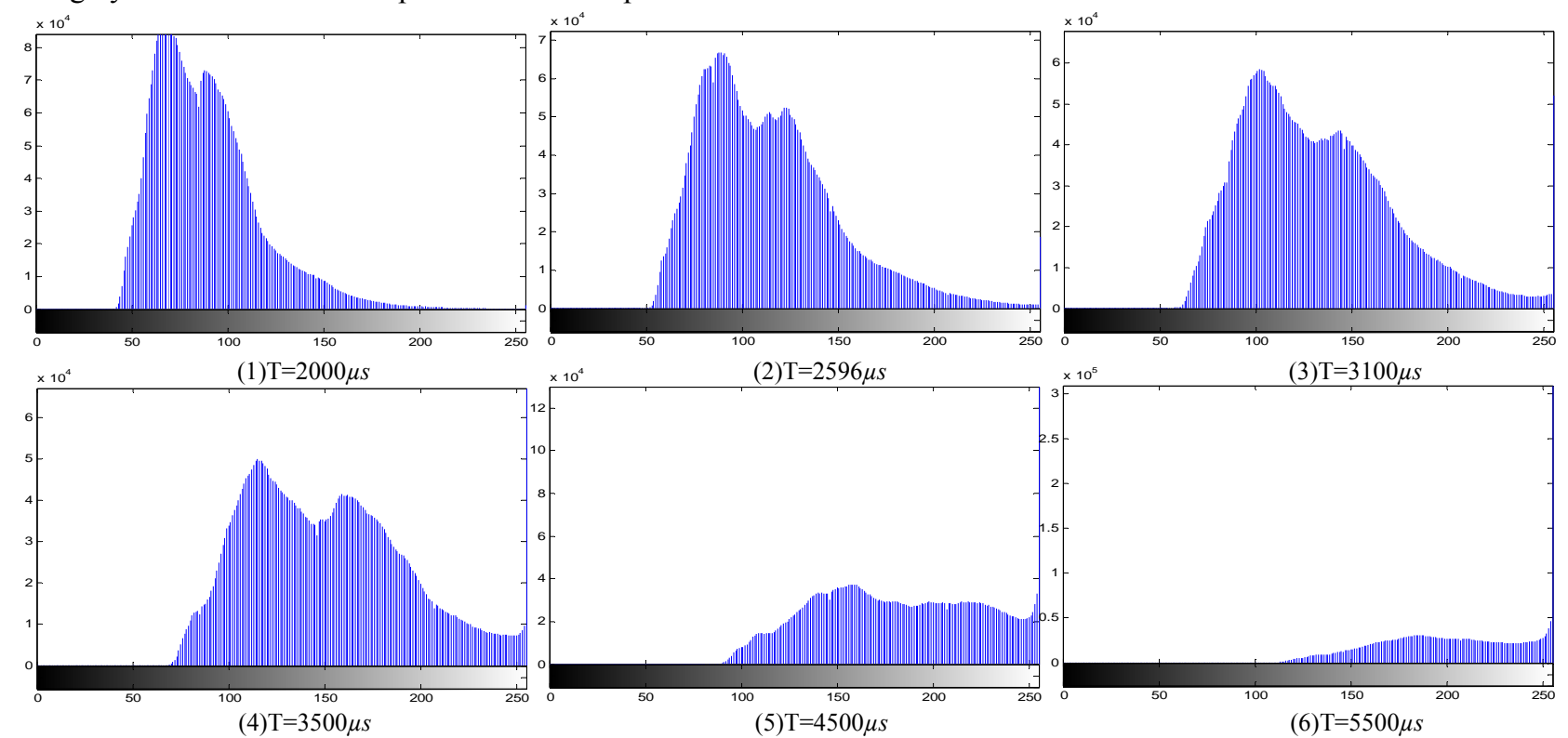

other methods. Therefore, the $3^{\text {rd }}$ moment about middle gray is valid to evaluate the exposure effect of the image whose histogram is unimodal.

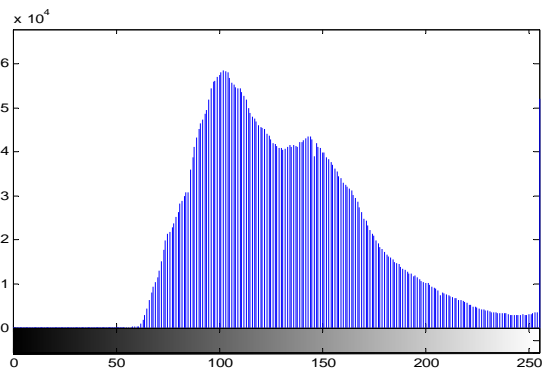

FIGURE II. GREEN COMPONENT HISTOGRAMS OF IMAGES IN FIGURE I

Above all, as the experimental result illustrate, the $3^{\text {rd }}$ moment about middle gray can be used to evaluate the exposure effect of the image whose histogram is unimodal.

\section{CONCLUSIONS}

In this paper, a new criterion for exposure evaluation is presented, which contributes in Auto Exposure control system. A new evaluation criterion is proposed based on the $3^{\text {rd }}$ moment about middle gray. Experimental result shows that the proposed evaluation criterion can be used to evaluate the exposure effect of the image whose histogram is unimodal.

\section{REFERENCES}

[1] T.Mori, "Automatic exposure control apparatus," US Patent $5703644,1997$.

[2] Inwook Shim, Joon-Young Lee, In So Kweon, "Auto-adjusting camera exposure for outdoor robotics using gradient information," 2014 IEEE/RSJ International Conference on Intelligent Robots and Systems. Chicago, pp.1011-1017. September 2014.

[3] Rahman MT, Kehtarnavaz N, Razlighi QR. "Using image entropy maximum for auto exposure,'J. Journal Of Electronic Imaging, vol. 20(1), 2011, pp.1917-1929.

[4] Liang Liang, Hung Bob, Noyes Ying, Velarde Ruben. "Real-time scene change detection assisted with camera $3 \mathrm{~A}$ (auto exposure, auto white balance, and auto focus)," Real-Time Image and Video Processing. San Francisco,vol.7871 pp.0F1-0F10, February 2011.

[5] Lee Cheol-Hee, Ha Yeongho. "Auto-exposure control using loop-up table based on scene-luminance curve in mobile phone camera," J. Signal Processing, vol.47(4), 2010, pp.56-62.

[6] Kang, Bongsoon. "Auto exposure control system using variable time constants," J. Journal of the Korea Institute Of Information and Communication Engineering, vol.11(2), 2007, pp.257-264.

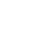

(1)

